\title{
ADI1, a methionine salvage pathway enzyme, is required for Drosophila fecundity
}

\author{
He-Yen Chou', Yu-Hung Lin', Guan-Lin Shiu', Hsiang-Yu Tang ${ }^{4,5}$, Mei-Ling Cheng ${ }^{4,5}$, Ming-Shi Shiao ${ }^{4,5}$ \\ and Li-Mei Pai ${ }^{1,2,3^{*}}$
}

\begin{abstract}
Background: Methionine, an essential amino acid, is required for protein synthesis and normal cell metabolism. The transmethylation pathway and methionine salvage pathway (MTA cycle) are two major pathways regulating methionine metabolism. Recently, methionine has been reported to play a key role in Drosophila fecundity.

Results: Here, we revealed that the MTA cycle plays a crucial role in Drosophila fecundity using the mutant of aci-reductone dioxygenase 1 (DADI1), an enzyme in the MTA cycle. In dietary restriction condition, the egg production of adil mutant flies was reduced compared to that of control flies. This fecundity defect in mutant flies was rescued by reintroduction of Dadi1 gene. Moreover, a functional homolog of human ADI1 also recovered the reproduction defect, in which the enzymatic activity of human ADI1 is required for normal fecundity. Importantly, methionine supply rescued the fecundity defect in Dadi1 mutant flies. The detailed analysis of Dadi1 mutant ovaries revealed a dramatic change in the levels of methionine metabolism. In addition, we found that three compounds namely, methionine, SAM and Methionine sulfoxide, respectively, may be required for normal fecundity.
\end{abstract}

Conclusions: In summary, these results suggest that ADI1, an MTA cycle enzyme, affects fly fecundity through the regulation of methionine metabolism.

Keywords: ADI1, Methionine, MTA cycle, Fecundity, Drosophila

\section{Background}

Methionine, one of the essential amino acids, plays a key role in protein synthesis and cellular metabolic function, supplying sulfur and other compounds required for normal metabolism and cell growth [1,2]. Methionine executes a well-known role for the initiation of protein synthesis in eukaryotes and prokaryotes. Indeed, the hydrophobic character of methionine is important for the binding of initiator tRNA to eIF-2, and most methionine residues are detected in the hydrophobic interior core of globular proteins [3,4]. Previous studies have also shown that methionine is a source of the methyl groups that regulate the methylation of DNA and histones, and influence chromatin structure and gene expression in the liver $[5,6]$. Methylation imbalance is correlated with several diseases including liver disease, cardiovascular disease and cancer $[7,8]$. Methionine

\footnotetext{
* Correspondence: pai@mail.cgu.edu.tw

${ }^{1}$ Graduate Institute of Biomedical Sciences, Chang Gung University, Tao-Yuan, Taiwan

${ }^{2}$ Department of Biochemistry, Chang Gung University, Tao-Yuan, Taiwan

Full list of author information is available at the end of the article
}

residues on the protein surface also function as endogenous antioxidants $[9,10]$. Recent study also indicated that methionine plays a critical role in fecundity in Drosophila [11].

Except from diet, methionine contents are controlled by several pathways, including the folate pathway, transmethylation pathway and methionine salvage pathway. The folate pathway plays a pivotal role in one-carbon metabolism $[12,13]$. In the transmethylation pathway, also called the methionine de novo pathway or methyl cycle, S-adenosylmethionine (SAM) is synthesized from ATP and methionine by SAM synthetase (SAM-S). The process of methyl cycle pathway is to metabolize SAM into S-adenosylhomocysteine ( $\mathrm{SAH}$ ) and subsequently to homocysteine. Then, homocysteine is converted into cysteine by trans-sulfuration or re-methylated to form methionine. SAM functions as a methyl donor involved in many biochemical reactions $[6,14]$. The methionine salvage pathway, also termed the 5 '-methylthioadenosine (MTA) cycle, is allowed to regenerate methionine from MTA and is also responsible for the production of 
polyamines which are critical for cell proliferation $[15,16]$. The biochemical reactions in the MTA cycle are mainly carried out by six enzymes which are conserved from bacteria to yeast to human $[17,18]$. However, the effects of the MTA cycle on methionine metabolism in Drosophila are still unknown.

In a previous study, Yeh et al. [19] identified Sip-L (hADI1), a hepatic factor capable of supporting HCV infection and replication in an otherwise non-permissive cell line. In a subsequent study, we demonstrated that human aci-reductone dioxygenase 1 (hADI1) over-expression in 293 cells enhances viral entry into cells but not replication of HCV [20]. ADI1, an MTA cycle enzyme, belongs to the cupin domain superfamily and has aci-reductone dioxygenase (ARD) enzymatic activity. The ADI1 associates with $\mathrm{Fe}^{2+}$ to produce formate and 2-keto-4-methylthiobutyrate (MTOB), the keto-acid precursor of methionine. Alternatively, ADI1 can associate with $\mathrm{Ni}^{2+}$ to produce formate, carbon monoxide and 3-methylthiopropionate $[21,22]$. Previous studies of hADI1 have shown that it has multiple functions, such as the modulation of cell migration, apoptosis and RNA processing [23-25]. Despite these functions in basic cellular processes, the roles of ADI1 in whole animals are unknown. Therefore, we attempted to investigate the function of ADI1 in model animal Drosophila

In the present study, we generated the Dadi1 null mutant and studied the role of Dadi1 in fly fecundity. We found that the enzymatic activity of ADI1 is required for normal egg production, and human ADI1 is functionally exchangeable for this effect. From the metabolomic analysis, we concluded that three metabolites in methionine metabolism might be critical for Drosophila fecundity.

\section{Methods}

\section{Fly strains}

Deletions in Dadi1 (CG32068) were generated by imprecise excision of P-element $\mathrm{P}\{\mathrm{XP}\} \mathrm{CG} 32068^{\mathrm{d} 01129}$ (Exelixis Collection), which is located at $48 \mathrm{bp}$ upstream of the start codon in exon 1 of the Dadi1 gene. First, the P-element strain was crossed with the rucuca strain (Bloomington stock number 576), which carries third-chromosome lines marked with several recessive markers. The recombined P-element strain was mobilized using standard genetic methods by crossing to Delta2-3/TM3,Sb (carrying the transposase) and excision alleles were identified by loss of white marker. Three null mutants (Dadi1 ${ }^{7}$, Dadi1 $^{9}$, Dadi $1^{74}$ ) with deletions in the Dadi1 gene and 11 hypomorphic alleles were selected from a collection of 508 excision strains by western blotting. Three null mutant alleles (Dadi1 ${ }^{7}$, Dadi $^{9}$, Dadi1 $\left.^{74}\right)$ also verified the deletion region in the Dadi1 gene by PCR amplification. The deletion region of $\mathrm{Dadi1}^{74}$ was from 10656504 to 10657550 in fly genomic DNA. The deletion region of Dadi1 and Dadi1 $^{9}$ were 10656504-10658482 and 1065542510657620, respectively. The lethality of Dadi1 null mutants (Dadi1 ${ }^{74}$ homozygote or Dadi $1^{7} /$ Dadi $^{9}$ transheterozygote) were shown to be less than $5 \%$.

To remove most of the markers in rucuca, the Dadi1 ${ }^{74}$ $\mathrm{FRT}^{80 \mathrm{~B}}$ strain was generated by crossing $D$ adi ${ }^{74}$ with $\mathrm{P}$ \{neoFRT\}80B/TM3Ser. The recombination was performed using standard genetics methods, and alleles were identified by containing two markers, thread (th) and ebony (e). The Dadi1 ${ }^{74}$-FRT ${ }^{80 \mathrm{~B}}$ strain was used in this study for the fecundity assay. The $\mathrm{P}\{$ neoFRT $\} 80 \mathrm{~B}$ strains were used in this paper for genetic background control.

To create the rescue construct P\{UAST-DADI1\}, first, Dadi1 cDNA encoding full-length DADI1 was amplified by PCR, inserted with hemagglutinin $(H A)$ epitope tag or without HA tag by EcoRI-XhoI sites, and introduced into the pBluescript (pBS) vector. Then, the EcoRI-XhoI fragment from $\mathrm{pBS}-\mathrm{HA}-D A D I 1$ and $\mathrm{pBS}-D A D I 1$ were subcloned into the pUAST vector. The constructs were injected into $w$ minus flies by P-element transformation. For the P\{UAST-hADI1 $\}$ and P\{UAST-hADI1-E94A $\}$ constructs, full-length human AD1 cDNA was amplified from the human liver cDNA. The strategy used for the hADI1 constructs was the same as the one used for the P\{UASTDADI1\} constructs. For the P\{UAST-hADI1-E94A\} construct, site-direct mutagenesis was performed in the pBS-hADI1 construct. The pBS-hADI1-E94A was subcloned into pUAST and then injected into the $w$ minus embryo.

\section{Western blotting}

For western blot analysis, fly adult samples were dissected and homogenized gently in cell lysate buffer (50 mMTris- $\mathrm{HCl}, \mathrm{pH} 7.5,400 \mathrm{mMNaCl}, 5 \mathrm{mM}$ EDTA, $1 \%$ Nonidet P-40 and protease inhibitor cocktail). Samples were left for $10 \mathrm{~min}$ on ice and then centrifuged at $14,000 \mathrm{rpm}$ for $10 \mathrm{~min}$ at $4^{\circ} \mathrm{C}$. The supernatant was then placed into a fresh centrifuge tube, protein sample buffer was added, and the sample was heated to $95^{\circ} \mathrm{C}$ for $10 \mathrm{~min}$; this was followed by analysis by 12\% SDSPAGE. The proteins were then transferred to PVDF membrane and incubated for $1 \mathrm{hr}$ in blocking buffer $(7 \%$ nonfat milk in TBS/0.1\% Tween-20). DADI1, hADI1or alpha-tubulin (Sigma) antibody incubations were carried out first in blocking buffer for $16 \mathrm{hr}$ at $4^{\circ} \mathrm{C}$ and then the membranes were washed with TBS/0.1\% Tween-20. HRPconjugated antibody was used as the secondary antibody for one hour. Finally, ECL substrate was added and protein signals were detected. For DADI1 antibody generation, DNA fragment-encoding full-length DADI1 was cleaved from pBS-DADI1, inserted by EcoRI-XhoI sites, and introduced into the pQE81L (QIAGEN) vector. DH5 $\alpha$ bacterial cells were transformed with $\mathrm{pQE} 81 \mathrm{~L}-\mathrm{DADI} 1$ and purified by Ni-NTA resin (QIAGEN). DADI1 polyclonal antibody was raised in rabbits and rats by immunization 
with His-tag-DADI1 fusion protein. The dilution of DADI1 antibody used for western blotting was 1:500. For hADI1 antibody generation, full-length hADI1 was amplified by PCR, inserted by EcoRI-XhoI sites, and introduced into the pET32a vector. The His-tag-hADI1 fusion proteins were overexpressed in BL21 bacterial cells and purified by Ni-NTA resin. Human ADI1 polyclonal antibody was raised in rabbits and the dilution of hADI1 antibody was 1:500.

\section{Food conditions}

For the fly food condition and fecundity assay, we used a protocol adapted from the one described in a study by Grandison et al. [11]. The restricted diet food contained $100 \mathrm{~g}$ BREWER'S Yeast, $50 \mathrm{~g}$ sucrose, $15 \mathrm{~g}$ agar, $3 \mathrm{ml}$ propionic acid and $30 \mathrm{ml} \mathrm{p}$-Hydroxy-benzoic acid methyl ester ( $3 \mathrm{~g}$ in $30 \mathrm{ml}$ 95\% ethanol) per liter. The fullyfed food was the same as the restricted diet food, except that the concentration of BREWER'S Yeast was increased to $200 \mathrm{~g}$ per liter. For methionine rescue experiments, the 0.7 or $1.4 \mathrm{mM}$ methionine (Sigma, M9625) or $0.4 \mathrm{mM}$ tryptophan (Sigma, T0254) was added to restricted diet food.

\section{Fecundity assay}

Homozygous mutant flies (Dadi1 ${ }^{74} / \operatorname{Dadi1}^{74}$ ) were generated by crossing male Dadi1 heterozygous mutant flies with female Dadi1 heterozygous mutant flies. Under the restricted diet condition, 135 Dadi1 homozygous mutant female flies were mixed with 90 OreR male flies to mate for two days in 5 egg laying cups. The females were then separated from males and transferred to new egg laying cups with the restricted diet food. The density of flies was 30 females per egg laying cup. At least 90 females per one genotype were observed in various experiments. We regularly transferred flies to new egg laying cups after approximately 2 days and collected eggs on the appointed days. The eggs were counted on days $3,6,8$, $11,15,22,29$ and 35, and a Student's $t$ test was used to investigate differences between the various genotypes.

\section{Targeted metabolites analysis}

The methionine-associated metabolites were determined from wild-type and Dadi1 homozygous mutant ovaries raised under the restricted diet condition. Twenty ovaries per genotype were collected and homogenized in $80 \% \mathrm{MeOH}$. The extraction samples were centrifuged at $12000 \mathrm{rpm}$ for $10 \mathrm{~min}$ at $4^{\circ} \mathrm{C}$. The supernatants were transferred into clean tubes, dried with $\mathrm{N} 2$, and stored at $-80^{\circ} \mathrm{C}$ until UPLC/MS analysis. Samples were subjected to Ultra performance liquid chromatography coupled with triple quadrupole massspectrometry (UPLC/TQMS) system. Results were further analyzed using the Masslyn $x^{\text {Tn }} 4.0$ and QuanLynx $^{\text {tw }}$ (Waters) software systems. The UPLC/
TQMS analysis was conducted in the Metabolomics Core Laboratory of the Healthy Aging Research Center, Chang Gung University.

\section{Results}

\section{The generation of Drosophila adi1 mutants}

To investigate the role of methionine metabolism in Drosophila fecundity, we generated Dadi1 null mutants, by imprecise excision of the $\mathrm{P}$ element from strain $\mathrm{P}$ \{XP\}CG32068d01129 (Exelixis Collection). The Drosophila adi1 gene encodes aci-reductone dioxygenase (ARD) and presumably functions in the MTA cycle (Figure 1A). It is located on chromosome $3 \mathrm{~L}$ at $67 \mathrm{E} 6$, and consists of five exons that encode a predicted protein of 186 amino acids (Flybase database ID CG32068). The P $\{X P\}$ CG32068 d01129 P-element is located at 48 bp upstream of the start codon, in exon 1 of the Dadil gene. Using genomic PCR amplification, three null mutants $\left(\right.$ Dadi1 $^{7}$, Dadi1 $^{9}$, Dadi1 $^{74}$ ) with deletions in the Dadi1 gene were selected from a collection of 508 excision strains (Figure 1B). The deleted region of Dadi1 $1^{74}$ includes an entire coding region of Dadi1 gene but has no effects on neighboring genes, while the deleted regions of $\mathrm{Dadi1}^{7}$ and $D$ adi1 ${ }^{9}$ are larger and include parts of the $5^{\prime}$ and 3' neighboring genes, respectively. Therefore, homozygote $\operatorname{Dadi1}^{74}$ and trans-heterozygote of $\operatorname{Dadi1}^{7} / \operatorname{Dadi1}^{9}$ served as null mutants. More than $95 \%$ of the Dadi1 null mutants eclosed to viable adults. Polyclonal antiDADI1 antibody raised against full-length protein, recognized one major band about $19 \mathrm{kDa}$ in immunoblots of wild-type extracts from different developmental stages (Figure 1C). The ubiquitous expression of DADI1 throughout all developmental stages suggested it could serve as an enzyme involved in metabolic reactions in fly life. A lack of proteins in null mutants was confirmed by a lack of signaling in western blotting for Dadil ${ }^{7}$ / Dadi $1^{9}$ trans-heterozygous and Dadi1 $1^{74}$ homozygous flies (Figure 1D). To our knowledge, the Dadi1 mutant is the first mutant involved in the MTA cycle in Drosophila. Thus, the Dadi1 mutant provides us with an opportunity to investigate the role of the MTA cycle in Drosophila development.

\section{Dadi1 mutant females displayed a fecundity defect under dietary restriction}

MTA cycle enzymes are actively expressed in Drosophila ovaries (Flybase). Thus, we examined fecundity, the egg production ability, was examined in control and Dadi $1^{74}$ mutant female flies under different food conditions. Since protein supply is a major factor for growth and dietary restriction is known to reduce fecundity in flies $[11,26,27]$, we designed both fully-fed and restricted diet conditions, which provided $20 \%$ and $10 \%$ yeast, respectively. Interestingly, under the restricted 


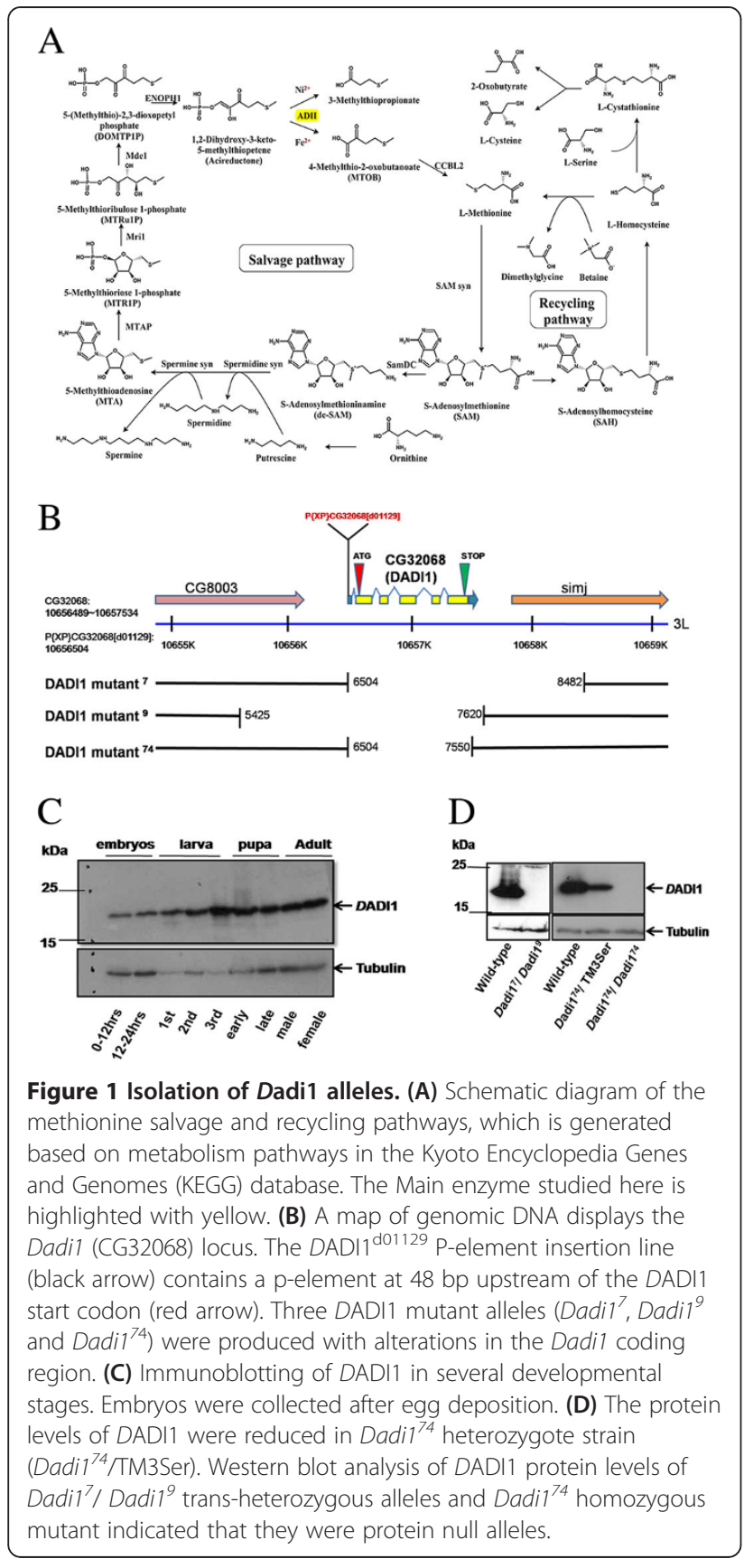

diet condition, Dadi1 $1^{74}$ mutant females displayed a significant reduction in fecundity compared to that in the control females (Figure 2A), indicating that Dadi1 is essential for fecundity when nutrition is poor. To verify whether this difference was caused by the lack of Dadi1, we expressed the exogenous Dadi1 gene in the mutant flies. The ubiquitous expression of DADI1 driven by actin-Gal4, indeed partially rescued the fecundity defect in Dadi1 mutant females (Figure 2B-C).
The enzymatic activity of Dadi1 is required for the regulation of fly fecundity

ADI1 family proteins are highly conserved among different species from bacteria to human [23], and hADI1 shared 50\% identity and 69\% similarity with DADI1 (CG32068) in protein sequence alignment (by NCBIblast). The enzyme activity of this protein family is located in the highly conserved ARD domain, and DADI1 showed $68 \%$ identity and $84 \%$ similarity to human ADI1 on this domain (Figure 3A). Previous studies have reported that human ADI1 obtains the ARD activity and functionally replaces yeast ADI1 in the in vivo enzyme activity assay [21]. Furthermore, a critical glutamic acid at the 94th residue has been demonstrated to be essential for its enzyme activity $[22,25]$. In this study, we generated transgenic flies expressing a wild type hADI1or a hADI1-E94A mutant in which the glutamic acid is substituted with alanine (Figure 3A-B). Indeed, both wild-type hADI1 strains almost completely rescued the fecundity defect in the Dadi1 mutants (Figure 3C). In contrast, hADI1-E94A could not rescue the fecundity defect in Dadi1 mutant females (Figure 3D), despite that the protein levels of E94A mutants and wild-type-15 of hADI1 were equal. Flies expressing this E94A mutant hADI1 displayed similar egg production ability to those expressing the GFP control protein. Based on these data, we concluded that human ADI1 is functionally interchangeable with Drosophila ADI1 and that the enzymatic activity of ADI1 is essential for normal egg production.

\section{Methionine supply suppressed the fecundity defect in Dadi1 mutant females}

The MTA cycle regulates the methionine metabolism. Thus we set out to test whether providing a supply of methinonine could rescue the fecundity defect in Dadi1 mutants. Indeed, adding $0.7 \mathrm{mM}$ or $1.4 \mathrm{mM}$ methionine to the fly food completely rescued the fecundity defect (Figure 4), such that the mutant females showed a similar ability to produce eggs to that of the control females. To further investigate whether the rescue was specifically due to methionine, tryptophan was also supplied in the food. However, no significant rescue in fecundity was found as a result of supplying tryptophan (Figure 4). Therefore, we concluded that the depletion of methionine was the major cause for reduced fecundity in Dadi1 mutants.

\section{Metabolites of MTA cycle were altered in Dadi1 mutant ovaries}

In order to understand what metabolites are critical for fly fecundity, we first examined the metabolism in Dadi1 mutant ovaries and in the ovaries from control flies in dietary restriction condition. UPLC-TQMS based targeted metabolites analysis indicated that metabolites in the MTA cycle, including methionine, SAM, MTA, and 

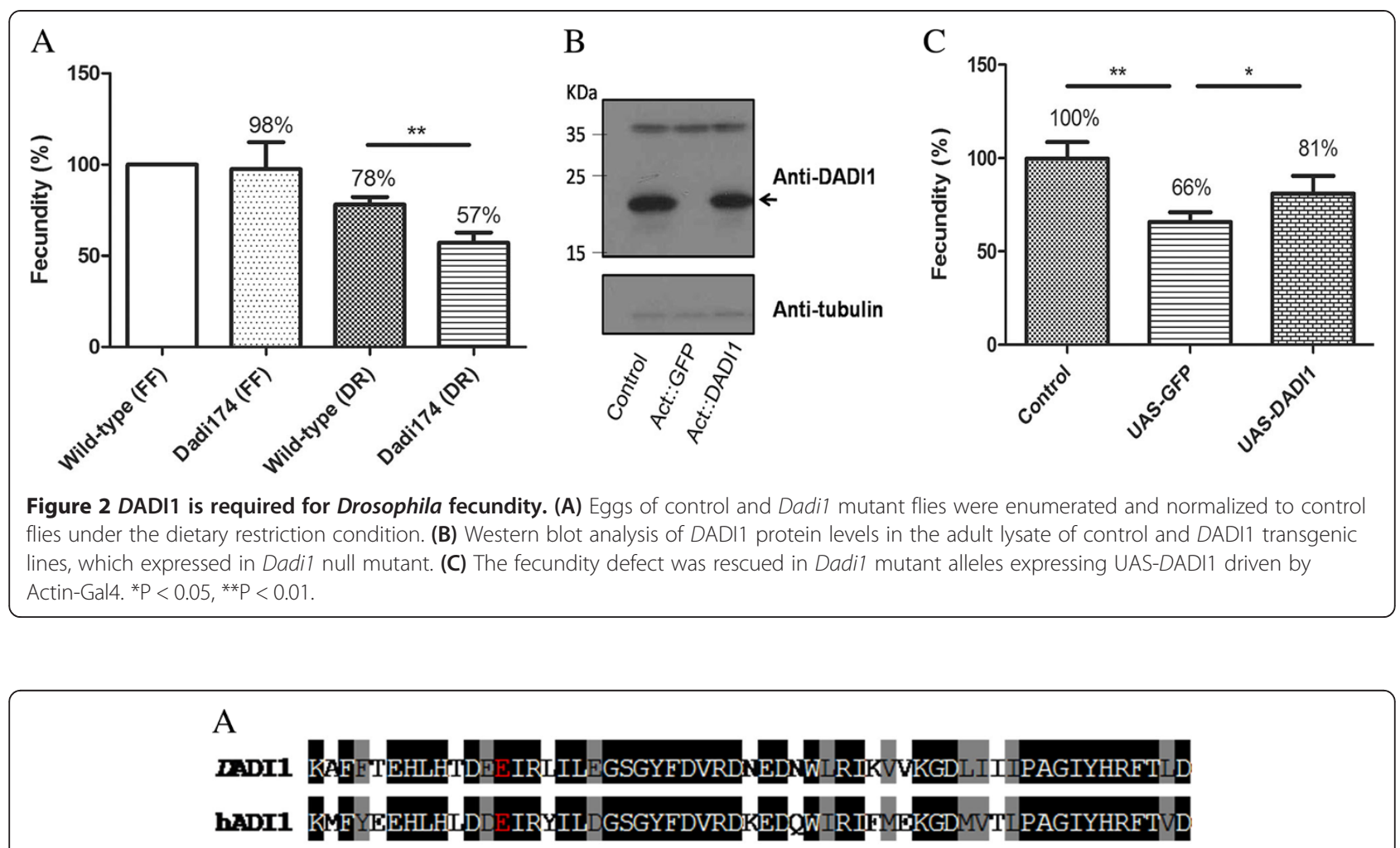

B $k$ Da
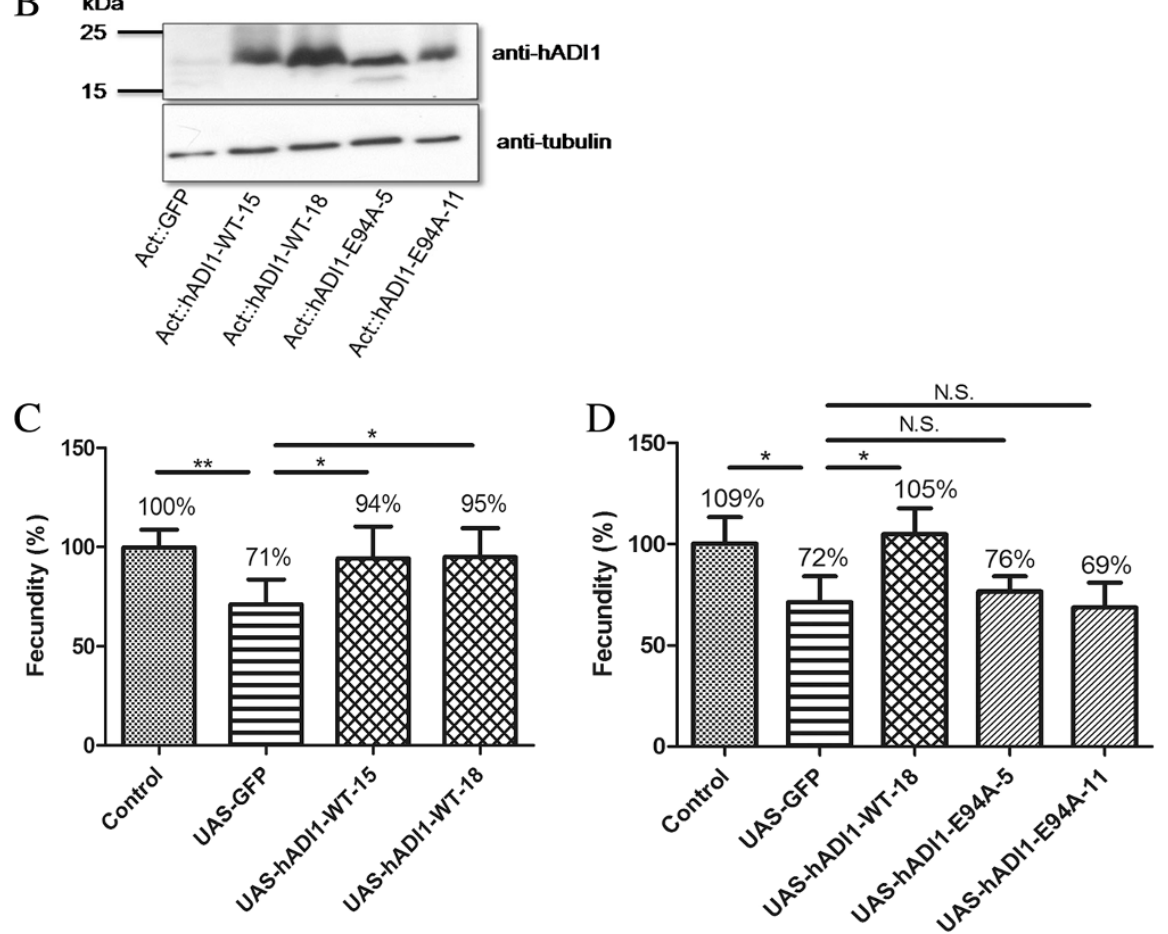

Figure 3 Enzymatic activity of ADI1 is essential for regulating egg production. (A) Protein sequence alignment is shown in the Cupin domain of hADI1 and DADI1. The identity and similarity of Cupin domain is $68 \%$ and $84 \%$, respectively. The glutamic acid site is required the ARD/ARD' family to execute enzymatic function (red star). (B) The protein expression levels of human wild-type and E94A mutant transgenic lines were observed in the Dadi1 null mutant background by hADI1 antibody. (C) Expressing human ADI1 by Actin-Gal4 can rescue the egg production defect in Dadi1 mutant alleles. (D) The fecundity was not rescued by diverse expressing enzyme dead mutant (E94A) transgenic lines. ${ }^{*} \mathrm{P}<0.05$, ${ }^{*}$ P $<0.01$ and N.S., no significance. 


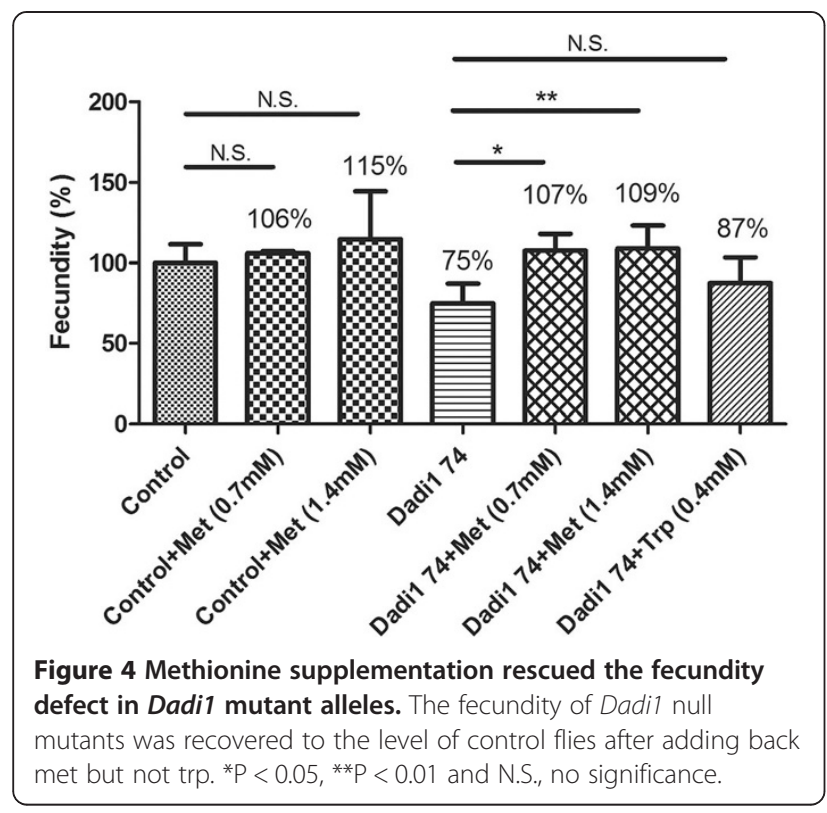

spermidine were significantly reduced. It clearly displayed that MTA cycle are significantly impaired in Dadi1 mutant ovaries (Figure 5A). Consistently, the metabolites in the downstream of ADI1 (methionine and SAM) were affected more severely than those upstream (MTA and spermidine). However, the metabolites in methyl cycle (SAH and homocysteine) and the trans-sulfuration (cysteine) were also affected, suggesting that under dietary restriction condition the methionine metabolism was greatly reduced in the Dadi1 mutant ovary (Figure 5B). The contents of Methionine sulfoxide, an oxidized form of methionine, were dramatically reduced. In contract, the levels of serine and phenylalanine, an indication of amino acid pools, remained unaffected (Figure 5B), suggesting that the general amino acid metabolism was not altered.

Since methionine supply in the diet rescued fecundity defect in Dadi1 mutant completely (Figure 4), we further compared the methionine metabolism in Dadi1 mutant ovaries and mutant ovaries with a methionine supply in the diet. Results demonstrated that the methionine supply elevated metabolites of MTA cycle, such as methionine, SAM, and MTA. Interestingly, the contents of spermidine were not increased. Nevertheless, metabolites in the methyl cycle and trans-sulfuration were not increased. These results suggest that MTA cycle might be dominant in methionine metabolism in fly ovary under dietary restriction condition. The amount of Methionine sulfoxide was also returned to the levels of control ovaries (Figure 5B). Again, the contents of serine and phenylalanine remain the same, indicating this amount of methionine in food supply did not alter general amino acid polls. Collectively, these results suggest that the rescue of fecundity by methionine most likely relates to the metabolites of the MTA cycle.
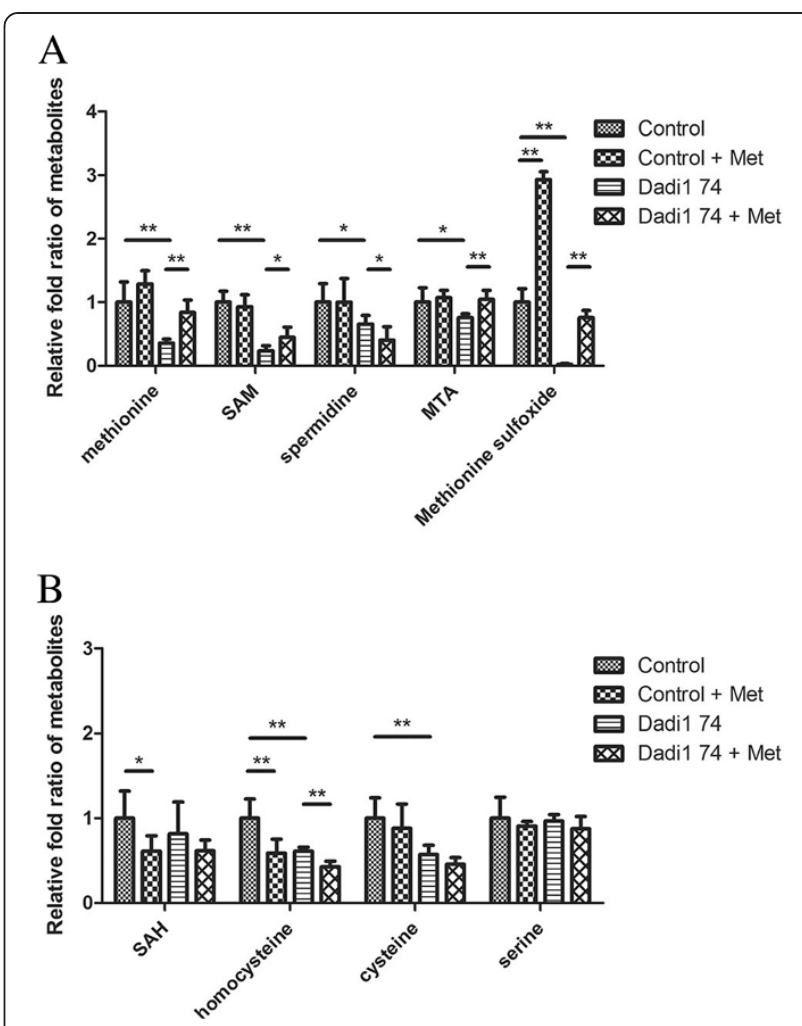

Figure 5 The metabolites of MTA cycle are affected by DADI1. (A) Four metabolites, Methionine, SAM, MTA and Methionine sulfoxide, were observed to be rescued in Dadi1 mutant ovaries by methionine addition. (B) Under the restricted diet condition, the metabolites of the methyl cycle and MTA cycle were dramatically changed in Dadil mutant ovaries. The raw data of metabolites are displayed below the picture. The results represent the means \pm SD of three experiments. ${ }^{*} P<0.05,{ }^{* * P}<0.01$ and N.S. indicates no significance.

\section{Discussion}

\section{Drosophila ADI1 is involved in MTA cycle}

In the present study, we demonstrated that the MTA cycle is crucial for Drosophila female fecundity under the restricted diet condition, and that a methionine supply could suppress the fecundity defect in Dadi1 mutants. The role of MTA cycle is used for the SAM, the principal methyl donor, which can recycle sulfur group to regenerate methionine. The MTA cycle also links tightly with polyamine synthesis. The intermediate compound of MTA cycle, MTA, is formed from dcSAM and a polyamine precursor [15]. In addition, the downstream products of MTA cycle, MTA and MTOB, are reported to inhibit the rate-limiting enzyme in polyamine synthesis, ornithine decarboxylase [28]. However, the biological functions of MTA cycle are still poorly understood. In previous studies, the MTA cycle has been found to process $10 \sim 15 \%$ of methionine contents in yeast [29], and is active in the human liver and kidney. Similarly, the MTA cycle is very active in Drosophila ovaries, which was revealed by the observation 
of a $64 \%$ reduction of methionine which could not be recycled back through the MTA cycle in Dadi1 mutant ovaries (Table 1).

\section{Drosophila fecundity is regulated by DADI1 through the alteration of methionine balance}

Drosophila female fecundity is controlled by nutrients intake and signaling pathways. For example, flies fed with restriction food can extend adult survivorship but reduce fecundity [30,31]. Moreover, scientists report that mutants in the insulin/insulin-like growth factors (IGFs) signaling pathway have reduced juvenile hormone contents, prolonged lifespan and impaired in reproduction [32-34]. Protein supply is considered very crucial for the growth and reproduction of organisms [11]. Recently, among all essential amino acids, methionine has been found to play the most pivotal role in Drosophila fecundity. Partridge et al. showed that adding methionine alone in restricted diet conditions food promoted longevity and increased fecundity [11]. When the maternal diets with an inappropriate intake of methionine can affect short-term reproductive ability and impair long-term health of the offspring [35]. A recent study suggested that the rat diet with low protein during gestation also observes the alterations of DNA methylation and gene expression in the offspring [36]. Furthermore, several studies also demonstrated that methionine is able to regulate gene expression and protein synthesis through the target of the rapamycin (mTOR) pathway $[37,38]$. The mTOR activity is modulated by the content of amino acids and insulin/insulin-like growth factors (IGFs). In contrast, adding methionine did not promote egg production in mutant flies that express a dominant- negative form of insulin receptor [11]. So, we propose that the MTA cycle may control methionine balance in Drosophila and lead to normal egg laying through the regulation of amino acid signals (via the mTOR pathway and insulin pathway). Further experiments are needed to reveal the details of how any mechanism controlled by methionine affects fecundity.

\section{The metabolites of methionine metabolism are affected} by DADI 1

Given that an increased methionine supply did not improve fecundity much in the control flies (Figure 4A), even as it increased the level of Methionine sulfoxide dramatically (by about 2 folds), we concluded that Methionine sulfoxide may not be the critical metabolite regulating fecundity. However, it is still possible that Methionine sulfoxide plays a role at the check-point of oogenesis progression. Therefore, when the content is below some threshold, the egg production is limited. This could explain why Methionine sulfoxide did not show a dosage effect on fecundity. In addition, spermidine, which is known to play a major role in cell proliferation, was not increased much with methionine supply (Figure 4B). Therefore, we hypothesized that the role of the MTA cycle in fly fecundity may not simply be to affect cell proliferation through control of spermidine contents. On the other hand, the content of spermidine in the fly ovary is much more than that required for normal fecundity, even under a restricted diet condition or in Dadi1 mutants. Based on the almost $100 \%$ recovery in metabolite contents, MTA may correlate with the rescued fecundity. MTA is known as a methyltransferase inhibitor, which influence methylation status [39]. Because the contents of MTA are very low in fly ovary, further experiments are needed to confirm the importance of MTA in fly fecundity. SAM is a universal methyl group donor for DNA, RNA, protein and lipid. In the fly ovary, SAM-S seems to be very active, leading to rapid conversion of methionine into SAM, such that SAM levels are 10 times greater than those of methionine. However, which of these three metabolites plays the most critical role and how it regulates fecundity are questions that remain to be resolved by further experiments.

\section{The relationship of reproduction and longevity is uncoupled in Dadi1 mutant allele}

Reduced fecundity has been found to be coupled with extended life spans in many organisms [26,40]. In Drosophila,

Table 1 The levels of biosynthetic metabolites

\begin{tabular}{lllll}
\hline Metabolites & Control & Control + Met & Dadi1-74 & $\begin{array}{c}\text { (ppm) } \\
\text { Dadi1-74 + Met }\end{array}$ \\
\hline SAM & $6.733 \pm 1.149$ & $6.217 \pm 1.295$ & $1.604 \pm 0.535$ & $3.014 \pm 1.061$ \\
Spermidine & $0.647 \pm 0.188$ & $0.647 \pm 0.241$ & $0.422 \pm 0.087$ & $0.262 \pm 0.134$ \\
MTA & $0.013 \pm 0.003$ & $0.014 \pm 0.002$ & $0.010 \pm 0.001$ & $0.014 \pm 0.002$ \\
Methionine sulfoxide & $15.852 \pm 3.377$ & $46.482 \pm 1.972$ & $0.392 \pm 0.206$ & $11.949 \pm 1.794$ \\
SAH & $0.028 \pm 0.009$ & $0.017 \pm 0.005$ & $0.023 \pm 0.011$ & $0.017 \pm 0.004$ \\
Homocysteine & $0.083 \pm 0.019$ & $0.049 \pm 0.014$ & $0.051 \pm 0.004$ & $0.035 \pm 0.005$ \\
Cysteine & $0.261 \pm 0.063$ & $0.230 \pm 0.075$ & $0.149 \pm 0.029$ & $0.119 \pm 0.021$ \\
Serine & $11.839 \pm 2.942$ & $10.746 \pm 0.643$ & $11.450 \pm 0.894$ & $10.374 \pm 1.722$ \\
\hline
\end{tabular}

The concentrations of metabolites were analyzed by UPLC-MS/MS and normalized with phenylalanine.

The unit of concentration is ppm (parts per million). 
protein supply played a pivotal role in female fecundity, and under the restricted diet condition fewer eggs were produced and the average life span was prolonged. Interestingly, adding back essential amino acids resulted in fecundity being recovered, whereas life span was reduced. However, supplying methionine appears to uncouple fecundity and life span, such that females lay normal numbers of eggs and while living just as long as control flies [11]. In Dadi1 mutants, the methionine content in ovaries was reduced to $36 \%$ of that in control flies, and the fecundity was diminished by about 20-30\% (Figures 2 and 3 and Table 1). However, Dadi1 mutants were less viable (5\% lethality) and had a shorter average life span (data not shown). It is possible that this reduced life span was caused by a metabolic imbalance. Consistently, the methionine supply did not alter the life span of Dadi1 mutants.

\section{Conclusions}

In summary, this study presents the first isolation of MTA cycle enzyme mutant alleles in Drosophila. The MTA cycle enzyme, DADI1, is required for normal fecundity. The fact that human ADI1 can rescue the egg production defect suggests that the functions of ADI1 proteins among different species are interchangeable and that the enzymatic activity of ADI1 is essential for its ability to regulate fly reproductive activity. The fecundity defect in Dadi1 mutants was rescued by adding methionine under dietary restriction. Using metabolic analysis, we found that DADI1 may regulate fly fecundity through the change of MTA cycle metabolites. The discovery of the Drosophila ADI1 protein in this study could clarify the role of the MTA cycle in methionine metabolism. Furthermore, the results of this study suggest that normal fecundity may be controlled by the metabolites of methionine metabolism.

\section{Competing interest}

The authors declare that they have no competing interests.

\section{Authors' contributions}

Conceived and designed the experiments: HYC, LMP and YHL; Performed experiments: HYC, YHL, GLS and HYT; Data analysis: HYC, YHL, MLC and MSS; Manuscript writing: HYC and LMP. All authors have read and approved the manuscript.

\section{Acknowledgements}

We thank Fly Core Taiwan, Bloomington Drosophila Stock Center, and Exelixis Collection at Harvard Medical School for fly stocks and reagents. We thank Drs Chien-Kuo Lee, Hai-Wei Pi and Chia-Lin Wu for their comments and the members of Pai lab for the valuable discussions. This work was supported by grants from the national Science Council of Taiwan, ROC (NSC100-2311-B-182001-MY3 to L.P.), the Chang Gung Memorial Hospital (CMRPD180111-3 to L.P.), and the Ministry of Education, Taiwan, ROC (EMRPD1C0041 to L.P.).

\section{Author details}

${ }^{1}$ Graduate Institute of Biomedical Sciences, Chang Gung University, Tao-Yuan, Taiwan. ${ }^{2}$ Department of Biochemistry, Chang Gung University, Tao-Yuan, Taiwan. ${ }^{3}$ Chang Gung Molecular Medicine Research Center, Chang Gung University, Tao-Yuan, Taiwan. ${ }^{4}$ Department of Biomedical Sciences, Chang Gung University, Tao-Yuan, Taiwan. ${ }^{5}$ Healthy Aging Research Center, College of Medicine, Chang Gung University, Tao-Yuan, Taiwan.
Received: 1 May 2014 Accepted: 8 July 2014

Published: 19 July 2014

\section{References}

1. Brosnan JT, Brosnan ME: The sulfur-containing amino acids: an overview. J Nutr 2006, 136:1636S-1640S.

2. Tesseraud S, Metayer Coustard S, Collin A, Seiliez I: Role of sulfur amino acids in controlling nutrient metabolism and cell functions: implications for nutrition. Br J Nutr 2009, 101:1132-1139.

3. Chao CC, Ma YS, Stadtman ER: Modification of protein surface hydrophobicity and methionine oxidation by oxidative systems. Proc Natl Acad Sci U S A 1997, 94:2969-2974.

4. Drabkin HJ, RajBhandary UL: Initiation of protein synthesis in mammalian cells with codons other than AUG and amino acids other than methionine. Mol Cell Biol 1998, 18:5140-5147.

5. Mato JM, Martinez-Chantar ML, Lu SC: S-adenosylmethionine metabolism and liver disease. Ann Hepatol 2013, 12:183-189.

6. Lu SC, Mato JM: S-adenosylmethionine in liver health, injury, and cancer. Physiol Rev 2012, 92:1515-1542.

7. Brosnan JT, da Silva R, Brosnan ME: Amino acids and the regulation of methyl balance in humans. Curr Opin Clin Nutr Metab Care 2007, 10:52-57.

8. Williams KT, Schalinske KL: New insights into the regulation of methyl group and homocysteine metabolism. J Nutr 2007, 137:311-314.

9. Levine RL, Mosoni L, Berlett BS, Stadtman ER: Methionine residues as endogenous antioxidants in proteins. Proc Natl Acad Sci U S A 1996, 93:15036-15040.

10. Kim G, Weiss SJ, Levine RL: Methionine oxidation and reduction in proteins. Biochim Biophys Acta 1840, 2014:901-905.

11. Grandison RC, Piper MD, Partridge L: Amino-acid imbalance explains extension of lifespan by dietary restriction in Drosophila. Nature 2009, 462:1061-1064.

12. Stipanuk MH: Sulfur amino acid metabolism: pathways for production and removal of homocysteine and cysteine. Annu Rev Nutr 2004, 24:539-577.

13. Stover PJ: Physiology of folate and vitamin B12 in health and disease. Nutr Rev 2004, 62:S3-S12. discussion S13.

14. Grillo MA, Colombatto S: S-adenosylmethionine and its products. Amino Acids 2008, 34:187-193.

15. Albers E: Metabolic characteristics and importance of the universal methionine salvage pathway recycling methionine from 5'-methylthioadenosine. IUBMB Life 2009, 61:1132-1142.

16. Kusano T, Berberich T, Tateda C, Takahashi Y: Polyamines: essential factors for growth and survival. Planta 2008, 228:367-381.

17. Sauter M, Moffatt $B$, Saechao MC, Hell R, Wirtz M: Methionine salvage and S-adenosylmethionine: essential links between sulfur, ethylene and polyamine biosynthesis. Biochem J 2013, 451:145-154.

18. Pirkov I, Norbeck J, Gustafsson L, Albers E: A complete inventory of all enzymes in the eukaryotic methionine salvage pathway. FEBS J 2008, 275:4111-4120.

19. Yeh CT, Lai HY, Chen TC, Chu CM, Liaw YF: Identification of a hepatic factor capable of supporting hepatitis $C$ virus replication in a nonpermissive cell line. J Virol 2001, 75:11017-11024.

20. Cheng JC, Yeh YJ, Pai LM, Chang ML, Yeh CT: 293 cells over-expressing human ADI1 and CD81 are permissive for serum-derived hepatitis C virus infection. J Med Virol 2009, 81:1560-1568.

21. Hirano W, Gotoh I, Uekita T, Seiki M: Membrane-type 1 matrix metalloproteinase cytoplasmic tail binding protein-1 (MTCBP-1) acts as an eukaryotic aci-reductone dioxygenase (ARD) in the methionine salvage pathway. Genes Cells 2005, 10:565-574.

22. Pochapsky TC, Pochapsky SS, Ju T, Mo H, Al-Mjeni F, Maroney MJ: Modeling and experiment yields the structure of acireductone dioxygenase from Klebsiella pneumoniae. Nat Struct Biol 2002, 9:966-972.

23. Uekita T, Gotoh I, Kinoshita T, Itoh Y, Sato H, Shiomi T, Okada Y, Seiki M: Membrane-type 1 matrix metalloproteinase cytoplasmic tail-binding protein-1 is a new member of the Cupin superfamily. A possible multifunctional protein acting as an invasion suppressor down-regulated in tumors. J Biol Chem 2004, 279:12734-12743.

24. Gotoh I, Uekita T, Seiki M: Regulated nucleo-cytoplasmic shuttling of human aci-reductone dioxygenase (hADI1) and its potential role in mRNA processing. Genes Cells 2007, 12:105-117.

25. Oram SW, Ai J, Pagani GM, Hitchens MR, Stern JA, Eggener S, Pins M, Xiao W, Cai X, Haleem R, Jiang F, Pochapsky TC, Hedstrom L, Wang Z: Expression and 
function of the human androgen-responsive gene ADI1 in prostate cancer. Neoplasia 2007, 9:643-651.

26. Flatt T: Survival costs of reproduction in Drosophila. Exp Gerontol 2011, 46:369-375.

27. Tu MP, Tatar M: Juvenile diet restriction and the aging and reproduction of adult Drosophila melanogaster. Aging Cell 2003, 2:327-333.

28. Subhi AL, Diegelman P, Porter CW, Tang B, Lu ZJ, Markham GD, Kruger WD: Methylthioadenosine phosphorylase regulates ornithine decarboxylase by production of downstream metabolites. J Biol Chem 2003, 278:49868-49873.

29. Chattopadhyay MK, Tabor CW, Tabor H: Methylthioadenosine and polyamine biosynthesis in a Saccharomyces cerevisiae meu1delta mutant. Biochem Biophys Res Commun 2006, 343:203-207.

30. Min K, Flatt T, Kulaots I, Tatar M: Counting calories in Drosophila diet restriction. Exp Gerontol 2007, 42:247-251.

31. Skorupa DA, Dervisefendic A, Zwiener J, Pletcher SD: Dietary composition specifies consumption, obesity, and lifespan in Drosophila melanogaster. Aging Cell 2008, 7:478-490.

32. Tatar M, Bartke A, Antebi A: The endocrine regulation of aging by insulin-like signals. Science 2003, 299:1346-1351.

33. Flatt T, Kawecki TJ: Juvenile hormone as a regulator of the trade-off between reproduction and life span in Drosophila melanogaster. Evolution 2007, 61:1980-1991

34. Toivonen JM, Partridge L: Endocrine regulation of aging and reproduction in Drosophila. Mol Cell Endocrinol 2009, 299:39-50.

35. Rees WD, Wilson FA, Maloney CA: Sulfur amino acid metabolism in pregnancy: the impact of methionine in the maternal diet. J Nutr 2006 , 136:1701S-1705S.

36. Lillycrop KA, Phillips ES, Jackson AA, Hanson MA, Burdge GC: Dietary protein restriction of pregnant rats induces and folic acid supplementation prevents epigenetic modification of hepatic gene expression in the offspring. J Nutr 2005, 135:1382-1386.

37. Stubbs AK, Wheelhouse NM, Lomax MA, Hazlerigg DG: Nutrient-hormone interaction in the ovine liver: methionine supply selectively modulates growth hormone-induced IGF-I gene expression. J Endocrinol 2002, 174:335-341.

38. Tesseraud S, Bigot $\mathrm{K}$, Taouis M: Amino acid availability regulates $\mathrm{S} 6 \mathrm{~K} 1$ and protein synthesis in avian insulin-insensitive QM7 myoblasts. FEBS Lett 2003, 540:176-180.

39. Avila MA, Garcia-Trevijano ER, Lu SC, Corrales FJ, Mato JM: Methylthioadenosine. Int J Biochem Cell Biol 2004, 36:2125-2130.

40. Flatt T: Ageing: Diet and longevity in the balance. Nature 2009, 462:989-990.

\section{doi:10.1186/s12929-014-0064-4}

Cite this article as: Chou et al:: ADI1, a methionine salvage pathway enzyme, is required for Drosophila fecundity. Journal of Biomedical Science 2014 21:64.

\section{Submit your next manuscript to BioMed Central and take full advantage of:}

- Convenient online submission

- Thorough peer review

- No space constraints or color figure charges

- Immediate publication on acceptance

- Inclusion in PubMed, CAS, Scopus and Google Scholar

- Research which is freely available for redistribution 